\title{
Time to Sustained Improvement in Bowel Movement Frequency with Telotristat Ethyl: Analyses of Phase III Studies in Carcinoid Syndrome
}

\author{
Joseph S. Dillon ${ }^{1}$ (D) - Matthew H. Kulke ${ }^{2}$ - Dieter Hörsch ${ }^{3}$ • Lowell B. Anthony ${ }^{4}$ • Richard R. P. Warner ${ }^{5}$. \\ Emily Bergsland $^{6} \cdot$ Staffan Welin ${ }^{7}$. Thomas M. O'Dorisio ${ }^{1}$. Pamela L. Kunz ${ }^{8} \cdot$ Chad McKee $^{9} \cdot$ Pablo Lapuerta $^{9}$. \\ Phillip Banks ${ }^{9} \cdot$ Marianne Pavel $^{10,11}$
}

Published online: 7 March 2020

(C) The Author(s) 2020

\begin{abstract}
Background Telotristat ethyl is approved to treat carcinoid syndrome diarrhea in combination with somatostatin analogs. In TELESTAR and TELECAST phase III studies, patients with carcinoid syndrome received telotristat ethyl 250 or $500 \mathrm{mg} 3$ times per day (tid) or placebo tid in addition to somatostatin analogs. The aim of this prespecified analysis was to examine the time to reductions in bowel movements (BMs) in the TELESTAR and TELECAST studies using survival analysis methods.

Methods First occurrence of sustained response was defined as the time to the first day of 2 consecutive weeks with a mean BM frequency improvement of $\geq 30 \%$ from baseline during the 12 -week double-blind treatment periods. Time to first $\geq 30 \%$ worsening in BM frequency was also measured. Treatments were compared with the log-rank test; Cox regression models provided point and confidence interval estimates of the hazard ratios for each trial.

Results In TELESTAR and TELECAST, majority of patients (69\%) on telotristat ethyl experienced a sustained $\geq 30 \%$ improvement in BM frequency. The median time to sustained reduction of at least $30 \%$ in BM frequency was significantly faster (fewer days to onset) for telotristat ethyl compared with placebo in both TELESTAR (250 mg, HR $=2.3$ [95\% CI, 1.3-4.1, $P=0.004$ ];
\end{abstract}

Sponsor: Lexicon Pharmaceuticals

Prior presentation of the manuscript: Selected data have been presented in abstract/poster format at the North American Neuroendocrine Tumor Society Symposium, Philadelphia, PA, USA, October 19-21, 2017 (Poster \#255), at the American Society of Clinical Oncology Gastrointestinal Cancers Symposium, San Francisco, CA, USA, January 18-20, 2018 (Poster \#395), and at the European Neuroendocrine Tumor Society Conference, Barcelona, Spain, March 7-9, 2018 (Poster \#J06).

Joseph S. Dillon

joseph-dillon@uiowa.edu

1 Department of Internal Medicine - Endocrinology and Metabolism, University of Iowa, Iowa City, IA, USA

2 Division of Medical Oncology, Boston Medical Center, Boston, MA, USA

3 Department of Gastroenterology/Endocrinology, Center for Neuroendocrine Tumors, Zentralklinik Bad Berka, Bad Berka, Germany

4 Division of Medical Oncology, University of Kentucky, Lexington, KY, USA

5 Division of Gastroenterology, Icahn School of Medicine at Mount Sinai, New York, NY, USA
6 Department of Medicine, University of California, San Francisco, San Francisco, CA, USA

7 Department of Endocrine Oncology, Uppsala University Hospital, Uppsala, Sweden

8 Department of Medicine - Division of Oncology, Stanford University, Palo Alto, CA, USA

9 Lexicon Pharmaceuticals, Inc., The Woodlands, TX, USA

10 Department of Hepatology and Gastroenterology, CharitéUniversitätsmedizin, Berlin, Germany

11 Department of Medicine 1, Division of Endocrinology, Friedrich-Alexander-Universität, Erlangen-Nürnberg, Erlangen, Germany 
$500 \mathrm{mg}, \mathrm{HR}=2.2$ [95\% CI, 1.2-3.9, $P=0.009]$ ) and TELECAST $(250 \mathrm{mg}, \mathrm{HR}=3.9$ [95\% CI, 1.6-11.1, $P=0.003] ; 500 \mathrm{mg}$, $\mathrm{HR}=4.2[95 \% \mathrm{CI}, 1.7-11.7, P=0.002])$. In TELECAST, $42 \%$ of patients on placebo experienced sustained worsening in BM frequency compared with $20 \%$ on telotristat ethyl; no significant difference was observed in TELESTAR.

Conclusion The time of onset of sustained BM frequency improvement mean and range are important when considering use of telotristat ethyl in patients with carcinoid syndrome diarrhea. Telotristat ethyl may also reduce sustained worsening in BM frequency.

Trial Registration ClinicalTrials.gov Identifiers: NCT01677910, NCT02063659

Keywords Malignant carcinoid syndrome $\cdot$ Tryptophan hydroxylase $\cdot$ Telotristat ethyl $\cdot$ Neuroendocrine tumors $\cdot$ Diarrhea Serotonin

\section{Introduction}

Patients with symptomatic carcinoid syndrome diarrhea can experience a high frequency of bowel movements (BMs), with one study reporting $5.0 \pm 2.7$ diarrhea episodes per day in 40 patients, prior to their initiation of somatostatin analog (SSA) therapy [1]. Diarrhea seriously impacts quality of life for these patients; $30 \%$ of patients will continue to have debilitating symptoms despite initiation of SSA therapy, while more will lose responsiveness to SSA therapy at indicated doses [2-4].

Telotristat ethyl is an oral tryptophan hydroxylase inhibitor recently approved by the United States (US) Food and Drug Administration (FDA) and European Medicines Agency/European Commission for the treatment of carcinoid syndrome diarrhea in combination with SSA therapy in adults whose symptoms are inadequately controlled by SSA therapy $[5,6]$. It has also been added to the US National Comprehensive Cancer Network (NCCN) guidelines for treatment of carcinoid syndrome-related diarrhea [7]. Its efficacy xand safety were evaluated in two phase III studies, TELESTAR and TELECAST, in patients with carcinoid syndrome. Significant reductions in BM frequency were demonstrated for patients taking telotristat ethyl in both studies $[8,9]$.

The pivotal, randomized phase III trial (TELESTAR) that resulted in FDA approval determined the efficacy of telotristat ethyl in reducing daily BM frequency from baseline $[5,8]$. Responses, predefined as a BM frequency reduction of $\geq 30 \%$ from baseline for $\geq 50 \%$ of the double-blind treatment (DBT) period, were observed in $44 \%$ and $42 \%$ of patients who received telotristat ethyl $250 \mathrm{mg}$ three times per day (tid) or telotristat ethyl $500 \mathrm{mg}$ tid, respectively, compared with only $20 \%$ of patients who received placebo [8].

SSAs are considered first-line therapy to treat neuroendocrine tumors (NETs) and carcinoid syndrome [10, 11]. The majority of study patients receiving telotristat ethyl in the two trials were also on stable doses of SSAs as standard of care $[8,9]$. SSAs improve carcinoid syndrome symptoms by interacting with extracellular receptors on NET cells, thereby regulating serotonin and other hormone release $[10,11]$. Telotristat ethyl improves carcinoid syndrome diarrhea by intracellular inhibition of tryptophan hydroxylase and blocks serotonin production within NET cells [12]. Although the medications are potentially complementary in treating carcinoid syndrome-related diarrhea, the underlying mechanisms differ, and the drugs may have different time courses of effect. Inaccurate expectations by patients and health care providers regarding the rapidity of onset of the effects of telotristat ethyl could lead to either inappropriate early discontinuation or prolonged use of medication. Thus, it is important to measure the onset of effect and potential for sustained improvement.

Although the main safety and efficacy results of telotristat ethyl use have already been published, the timing of responses has not been addressed in detail [8, 9]. Therefore, the objective of this prespecified analysis was to examine the time to achievement of BM frequency reductions in the TELESTAR and TELECAST studies using survival analysis methods. Median times to achievement of effect on related gastrointestinal symptoms, including changes in urgency and stool form, were also estimated.

\section{Methods}

The designs of the TELESTAR and TELECAST studies, which differ only in inclusion criteria, have been detailed elsewhere $[8,9]$. Briefly, patients with a history of welldifferentiated NETs were eligible for TELESTAR if they had a mean of $\geq 4$ BMs per day while on stable-dose SSA therapy. Patients were eligible for TELECAST, which was intended for those not qualifying for TELESTAR, if they had $<4$ BMs per day during the screening period and were receiving SSA therapy. Additionally, in the TELECAST study, patients with $\geq 4$ BMs per day were eligible if they were not receiving SSA therapy. All patients in TELECAST had carcinoid syndrome that required additional treatment, primarily owing to gastrointestinal symptoms. 
In both studies, baseline BM frequency was assessed with electronic diaries over a 3- to 4-week screening period. Qualifying patients were then randomized to placebo tid, telotristat ethyl $250 \mathrm{mg}$ tid, or telotristat ethyl $500 \mathrm{mg}$ tid (while continuing stable-dose SSA therapy, if already receiving SSA). Therapy was administered in a double-blinded fashion for 12 weeks. In the 500-mg tid dosage arm, there was blinded titration, with patients receiving $250 \mathrm{mg}$ tid during the first week. After the completion of the 12-week DBT period, all patients were offered open-label telotristat ethyl $500 \mathrm{mg}$ tid.

The primary endpoint in the TELESTAR study was the change from baseline in BM frequency, averaged over 12 weeks. The TELECAST study had co-primary endpoints of safety and changes in urinary 5-hydroxyindoleacetic acid (5-HIAA). Changes in BM frequency were a secondary endpoint in TELECAST and were analyzed in the same way as in TELESTAR.

The development of Kaplan-Meier curves for sustained reduction in BM frequency was prespecified in both studies. Sustained reduction was defined as the time from the first double-blind dose to the first day of two consecutive weeks (i.e., 14 days) with BM frequency of at least $30 \%$ below the individual baseline mean.

The $30 \%$ threshold was selected based on prior literature on BM frequency and patient-reported outcomes in patients with NETs [2]. Further support of this threshold came from patient interviews conducted among participants in a phase II study of telotristat ethyl and from an expert panel of physicians reviewing these data and their own experience [13]. The log-rank test was applied for both studies to compare the time to reduction in BM frequency among treatment arms.

Prespecified analyses also addressed the time to sustained worsening of BM frequency, using the same $30 \%$ threshold. This was prespecified for the TELESTAR and TELECAST studies because in a phase II study of telotristat ethyl, there was the suggestion of worsening of BM frequency observed on placebo [14].

In the TELESTAR and TELECAST studies, urgency to defecate was recorded daily on electronic diaries through a yes/no question. Stool form was also measured daily with the Bristol Stool Form 7-point scale $[15,16]$. Mean changes in stool form have been previously presented for TELESTAR and TELECAST [8, 9], whereas mean changes in urgency to defecate have been reported for TELESTAR and collected for TELECAST $[8,17]$. Differences between treatment groups were examined using least squares means. Differences were also examined at each week among patients who achieved $\geq$ $30 \%$ reductions in $\mathrm{BM}$ frequency, those who achieved $0-30 \%$ reductions in BM frequency, and those who had worsened BM frequency.
No adjustment for multiplicity was performed, as these analyses were intended to further qualify changes in BM frequency, which was already a primary endpoint in TELESTAR and a secondary endpoint in TELECAST.

\section{Results}

Baseline characteristics of patients in the TELESTAR and TELECAST studies are presented in Table 1. The key difference between the studies was the inclusion criteria for baseline BM frequency. In TELESTAR, the overall mean (SD) baseline BM frequency was 5.7 (1.8) BMs per day (range 3.511.5) and in TELECAST, it was 2.5 BMs per day (range 0.88-5.53). A total of 211 patients were treated in these 2 trials: 71 patients were randomized to receive placebo, 70 to telotristat ethyl $250 \mathrm{mg}$ tid, and 70 to telotristat ethyl $500 \mathrm{mg}$ tid; all patients were on SSA therapy, except for three patients in the telotristat ethyl $250 \mathrm{mg}$ tid arm and five patients in the telotristat ethyl $500 \mathrm{mg}$ tid arm in the TELECAST study [8, 9].

Significant BM frequency reductions with telotristat ethyl compared with placebo were seen in both studies, as were significant reductions in BM frequency from baseline [8, 9]. At week 12 in the TELESTAR study, the mean (SD) BM frequency changes from baseline were $-0.9(1.2),-1.7$ (1.7), and - 2.1 (1.9) BMs per day on placebo (SSA alone), telotristat ethyl $250 \mathrm{mg}$ tid, and telotristat ethyl $500 \mathrm{mg}$ tid, respectively ( $p<0.001$ for each compared with baseline) [8]. At week 12 in the TELECAST study, the mean (SD) BM frequency changes from baseline were $+0.06(0.5),-0.79$ (1.0), and -0.85 (1.0) BMs per day on placebo $(p=0.44$, compared with baseline), telotristat ethyl $250 \mathrm{mg}$ tid ( $p=$ 0.008 , compared with baseline), and telotristat ethyl $500 \mathrm{mg}$ tid ( $p<0.001$, compared with baseline), respectively [9].

The median time to sustained reduction of at least $30 \%$ in BM frequency was significantly faster (fewer days to onset) for telotristat ethyl compared with placebo in both TELESTAR (Fig. 1a and Table 2) and TELECAST (Fig. 1b and Table 2). In TELESTAR, the median times to first occurrence of sustained $\geq 30 \%$ improvement were 19 days and 27 days for the telotristat ethyl $250 \mathrm{mg}$ tid $(\mathrm{HR}=2.3,95 \%$ CI, 1.3-4.1, $p=0.004$ ) and telotristat ethyl $500 \mathrm{mg}$ tid treatment groups ( $\mathrm{HR}=2.2,95 \% \mathrm{CI}, 1.2-3.9, p=0.009)$, respectively ( $n=45$ for both groups). In TELECAST, the median times to improvement were 34 days and 39 days for the telotristat ethyl $250 \mathrm{mg}$ tid $(\mathrm{HR}=3.9,95 \% \mathrm{CI}, 1.6-11.0$, $p=0.003)$ and telotristat ethyl $500 \mathrm{mg}$ tid groups $(\mathrm{HR}=4.2$, 95\% CI, 1.7-11.7, $p=0.002$ ), respectively ( $n=25$ for both groups). Median time to sustained improvement was not reached on placebo in either study.

The timing of response varied widely among patients. In TELESTAR, the earliest sustained improvement in BM frequency was observed at three days of telotristat ethyl 
Table 1 Baseline characteristics

\begin{tabular}{|c|c|c|c|c|c|c|c|}
\hline \multirow[b]{2}{*}{ Characteristic } & \multirow[b]{2}{*}{ Statistic } & \multicolumn{3}{|l|}{ TELESTAR } & \multicolumn{3}{|l|}{ TELECAST } \\
\hline & & Placebo $(N=45)$ & $\begin{array}{l}\text { Telotristat ethyl } \\
250 \mathrm{mg}(N=45)\end{array}$ & $\begin{array}{l}\text { Telotristat ethyl } \\
500 \mathrm{mg}(N=45)\end{array}$ & Placebo $(N=26)$ & $\begin{array}{l}\text { Telotristat ethyl } \\
250 \mathrm{mg}(N=25)\end{array}$ & $\begin{array}{l}\text { Telotristat ethyl } \\
500 \mathrm{mg}(N=25)\end{array}$ \\
\hline \multirow[t]{2}{*}{ u5-HIAA (mg per 24 h) } & $n$ & 44 & 42 & 44 & 26 & 25 & 25 \\
\hline & Mean (SD) & $80.97(161.01)$ & $92.65(114.90)$ & $89.50(144.47)$ & $81.99(113.61)$ & $86.30(73.51)$ & $66.05(88.94)$ \\
\hline \multirow[t]{2}{*}{ BMs (counts per day) } & $n$ & 45 & 45 & 45 & 25 & 25 & 25 \\
\hline & Mean (SD) & $5.20(1.35)$ & $6.09(2.07)$ & $5.81(1.96)$ & $2.19(0.67)$ & $2.53(1.25)$ & $2.79(1.57)$ \\
\hline \multirow[t]{2}{*}{ Stool form score ${ }^{a}$} & $n$ & 45 & 45 & 45 & 25 & 25 & 25 \\
\hline & Mean (SD) & $5.92(0.70)$ & $5.93(0.50)$ & $5.97(0.69)$ & $4.97(0.91)$ & $5.11(0.84)$ & $5.29(0.83)$ \\
\hline \multirow{2}{*}{$\begin{array}{l}\text { Urgency/immediate } \\
\text { need to defecate } \\
\text { (proportion of days) }\end{array}$} & $n$ & 38 & 39 & 38 & 26 & 25 & 25 \\
\hline & Mean (SD) & $0.88(0.16)$ & $0.84(0.24)$ & $0.85(0.27)$ & $0.40(0.37)$ & $0.29(0.28)$ & $0.44(0.37)$ \\
\hline
\end{tabular}

Abbreviations: $B M$, bowel movement; $S D$, standard deviation; $u$ 5-HIAA, urinary 5-hydroxyindoleacetic acid

${ }^{\text {a }}$ Bristol Stool Form Scale [16]

treatment, and the latest was at 73 days. In TELECAST, the earliest sustained improvement in BM frequency was observed at four days of telotristat ethyl treatment, and the latest was at 68 days. In TELESTAR, hazard ratios for sustained reduction of at least $30 \%$ in $\mathrm{BM}$ frequency were $2.3(95 \% \mathrm{CI}, 1.3-4.1)$ and $2.2(95 \%$
Fig. 1 Kaplan-Meier curves for improvement in BM frequency. Probability with time that a patient would not have a sustained $\geq 30 \%$ improvement in BM frequency for patients in the TELESTAR (a) and TELECAST (b) studies. A sustained $\geq 30 \%$ improvement from baseline was defined as the time from the first DBT dose date to the first day of a consecutive 14-day period with BM frequency at least $30 \%$ below the individual baseline BM frequency. Patients having no $\geq 30 \%$ improvement were censored on the last day of the last week a valid assessment of the endpoint can be made. Shapes indicate censored values. Abbreviations: BM, bowel movement; DBT, double-blind treatment; tid, three times per day
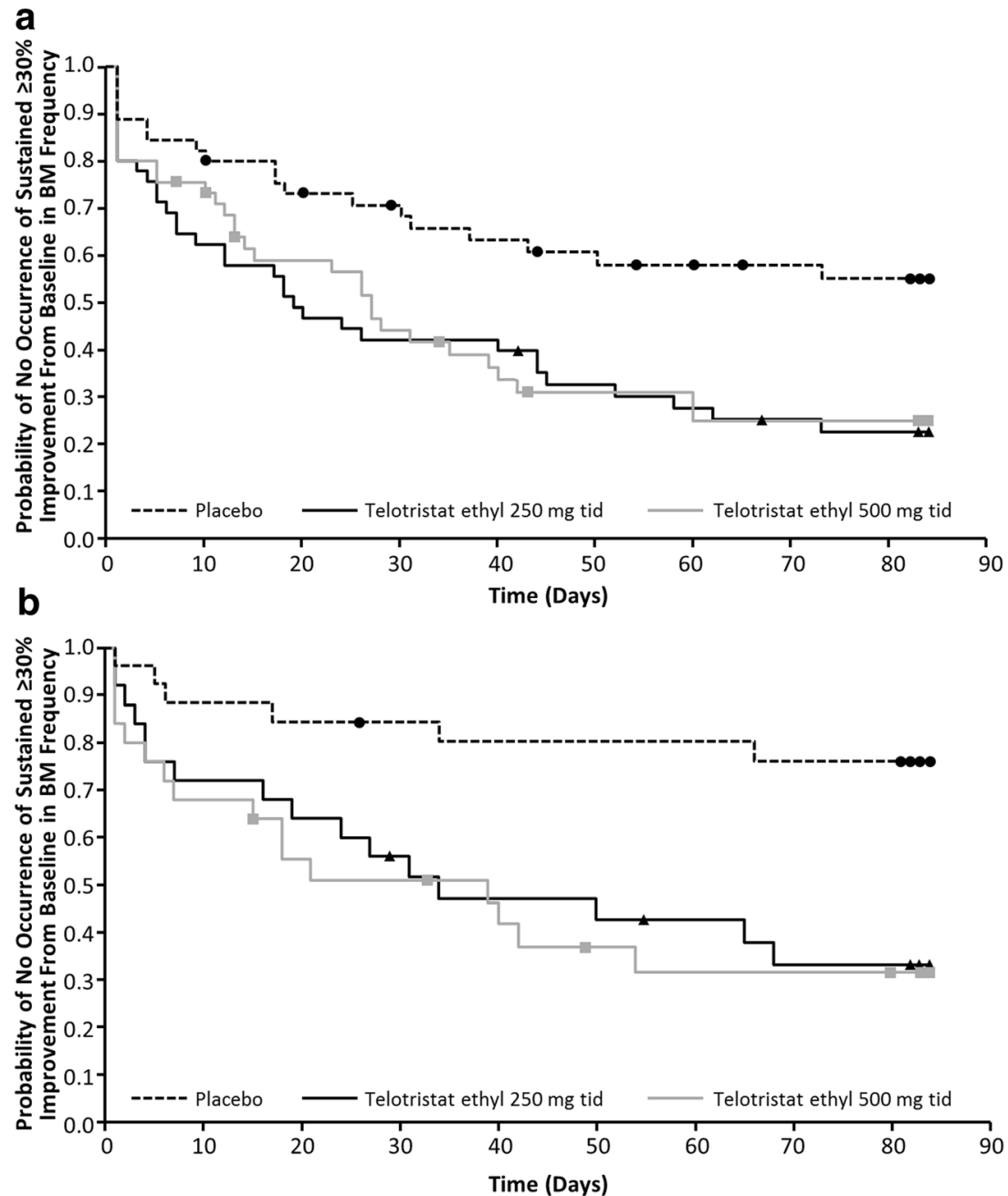
Table 2 Number of days to first occurrence of a sustained $\geq 30 \%$ improvement from baseline in BM frequency during the DBT period

\begin{tabular}{|c|c|c|c|c|c|c|}
\hline \multirow[b]{2}{*}{ Statistic } & \multicolumn{3}{|l|}{ TELESTAR } & \multicolumn{3}{|c|}{ TELECAST } \\
\hline & Placebo $(n=45)$ & $\begin{array}{l}\text { Telotristat ethyl } \\
250 \mathrm{mg}(n=45)\end{array}$ & $\begin{array}{l}\text { Telotristat ethyl } \\
500 \mathrm{mg}(n=45)\end{array}$ & $\begin{array}{l}\text { Placebo } \\
(n=26)\end{array}$ & $\begin{array}{l}\text { Telotristat ethyl } \\
250 \mathrm{mg}(n=25)\end{array}$ & $\begin{array}{l}\text { Telotristat ethyl } \\
500 \mathrm{mg}(n=25)\end{array}$ \\
\hline Patient with an event, $n$ & 19 & 34 & 31 & 6 & 16 & 16 \\
\hline Censored, $n$ & 26 & 11 & 14 & 20 & 9 & 9 \\
\hline $\begin{array}{l}\text { 25th percentile } \\
\quad(95 \% \mathrm{CL})^{\mathrm{a}} \text {, days }\end{array}$ & $18.0(4.0,50.0)$ & $5.0(1.0,12.0)$ & $10.0(1.0,15.0)$ & NR & $7.0(3.0,31.0)$ & $6.0(1.0,21.0)$ \\
\hline $\begin{array}{l}\text { Median percentile } \\
\quad(95 \% \mathrm{CL})^{\mathrm{a}} \text {, days }\end{array}$ & NR & $19.0(9.0,44.0)$ & $27.0(13.0,40.0)$ & NR & $34.0(\mathrm{NC})$ & $39.0(\mathrm{NC})$ \\
\hline $\begin{array}{l}\text { 75th percentile } \\
\quad(95 \% \mathrm{CL})^{\mathrm{a}} \text {, days }\end{array}$ & NR & $73.0(\mathrm{NC})$ & NR & NR & NR & NR \\
\hline $\begin{array}{l}\text { HR (95\%), telotristat } \\
\text { ethyl vs placebo }\end{array}$ & - & $2.3(1.3,4.1)$ & $2.2(1.2,3.9)$ & - & $3.9(1.6,11.0)$ & $4.2(1.7,11.7)$ \\
\hline$p$ value $^{\mathrm{c}}$ & - & 0.004 & 0.009 & - & 0.003 & 0.002 \\
\hline
\end{tabular}

Abbreviations: $B M$, bowel movement; $C L$, confidence limits; $D B T$, double-blind treatment; $H R$, hazard ratio; $N C$, not calculated; $N R$, not reached; $u 5$ HIAA, urinary 5-hydroxyindoleacetic acid

${ }^{a}$ Median, percentiles, and 95\% CL are from Kaplan-Meier estimates.

${ }^{\mathrm{b}} \mathrm{HR}$ and $95 \% \mathrm{CL}$ are from Cox proportional hazards regression model, which includes treatment group and u5-HIAA stratification at randomization as fixed effects.

${ }^{\mathrm{c}} \mathrm{p}$-value is from log-rank test stratified by $\mathrm{u} 5$-HIAA at randomization.

CI, 1.3-3.9) for telotristat ethyl $250 \mathrm{mg}$ tid and $500 \mathrm{mg}$ tid, respectively. In TELECAST, the hazard ratios for sustained reduction of at least $30 \%$ in BM frequency were 3.9 (95\% CI, 1.6-11.0) and 4.2 (95\% CI, 1.711.7) for telotristat ethyl $250 \mathrm{mg}$ tid and $500 \mathrm{mg}$ tid, respectively ( $p \leq 0.003$, for each comparison versus placebo).
Time to worsening of BM frequency was also measured for both trials (Table 3 ). In TELESTAR, there were very few patients with sustained worsening: 2,2 , and 0 patients with sustained worsening on placebo, telotristat ethyl $250 \mathrm{mg}$ tid, and telotristat ethyl $500 \mathrm{mg}$ tid, respectively, with no statistically significant difference between telotristat ethyl and placebo for either treatment arm (Fig. 2a).

Table 3 Number of days to first occurrence of $\geq 30 \%$ worsening from baseline in BM frequency during the DBT period

\begin{tabular}{|c|c|c|c|c|c|c|}
\hline \multirow[b]{2}{*}{ Statistic } & \multicolumn{3}{|l|}{ TELESTAR } & \multicolumn{3}{|l|}{ TELECAST } \\
\hline & Placebo $(n=45)$ & $\begin{array}{l}\text { Telotristat ethyl } \\
250 \mathrm{mg}(n=45)\end{array}$ & $\begin{array}{l}\text { Telotristat ethyl } \\
500 \mathrm{mg}(n=45)\end{array}$ & Placebo $(n=26)$ & $\begin{array}{l}\text { Telotristat ethyl } \\
250 \mathrm{mg}(n=25)\end{array}$ & $\begin{array}{l}\text { Telotristat ethyl } \\
500 \mathrm{mg}(n=25)\end{array}$ \\
\hline Patient with an event, $n$ & 2 & 2 & 0 & 11 & 7 & 3 \\
\hline Censored, $n$ & 43 & 43 & 45 & 15 & 18 & 22 \\
\hline $\begin{array}{l}\text { 25th percentile } \\
\qquad(95 \% \mathrm{CL})^{\mathrm{a}} \text {, days }\end{array}$ & NR & NR & NR & $21.0(\mathrm{NC})$ & $52.0(\mathrm{NC})$ & NR \\
\hline $\begin{array}{l}\text { Median percentile } \\
(95 \% \mathrm{CL})^{\mathrm{a}} \text {, days }\end{array}$ & NR & NR & NR & NR & NR & NR \\
\hline $\begin{array}{l}\text { 75th percentile } \\
\qquad(95 \% \mathrm{CL})^{\mathrm{a}} \text {, days }\end{array}$ & NR & NR & NR & NR & NR & NR \\
\hline $\begin{array}{l}\text { HR (95\%), telotristat } \\
\text { ethyl vs placebo }\end{array}$ & - & $1.0(0.1,8.4)$ & $0.0(\mathrm{NC})$ & - & $0.5(0.2,1.4)$ & $0.3(0.1,0.8)$ \\
\hline$p$ value $\mathrm{e}^{\mathrm{c}}$ & - & $>0.99$ & 0.18 & - & 0.18 & 0.021 \\
\hline
\end{tabular}

Abbreviations: $B M$, bowel movement; $C L$, confidence limits; $D B T$, double-blind treatment; $H R$, hazard ratio; $N C$, not calculated; $N R$, not reached; $u 5$ HIAA, urinary 5-hydroxyindoleacetic acid

${ }^{a}$ Median, percentiles, and 95\% CL are from Kaplan-Meier estimates.

${ }^{\mathrm{b}} \mathrm{HR}$ and $95 \% \mathrm{CL}$ are from Cox proportional hazards regression model, which includes treatment group and u5-HIAA stratification at randomization as fixed effects

${ }^{\mathrm{c}} \mathrm{p}$-value is from log-rank test stratified by $\mathrm{u} 5$-HIAA at randomization. 
Fig. 2 Kaplan-Meier curves for worsening in BM frequency. Probability with time that a patient would not have $\mathrm{a} \geq 30 \%$ worsening in BM frequency for patients participating in the TELESTAR (a) and TELECAST (b) studies. The time to $\geq 30 \%$ worsening from baseline was defined as the time from the first DBT dose date to the first day of a consecutive 14-day period with BM frequency at least $30 \%$ above the individual baseline BM frequency. Patients having no $\geq 30 \%$ worsening were censored on the last day of the last week a valid assessment of the endpoint can be made. Shapes indicate censored values. Abbreviations: BM, bowel movement; DBT, double-blind treatment; tid, three times per day a

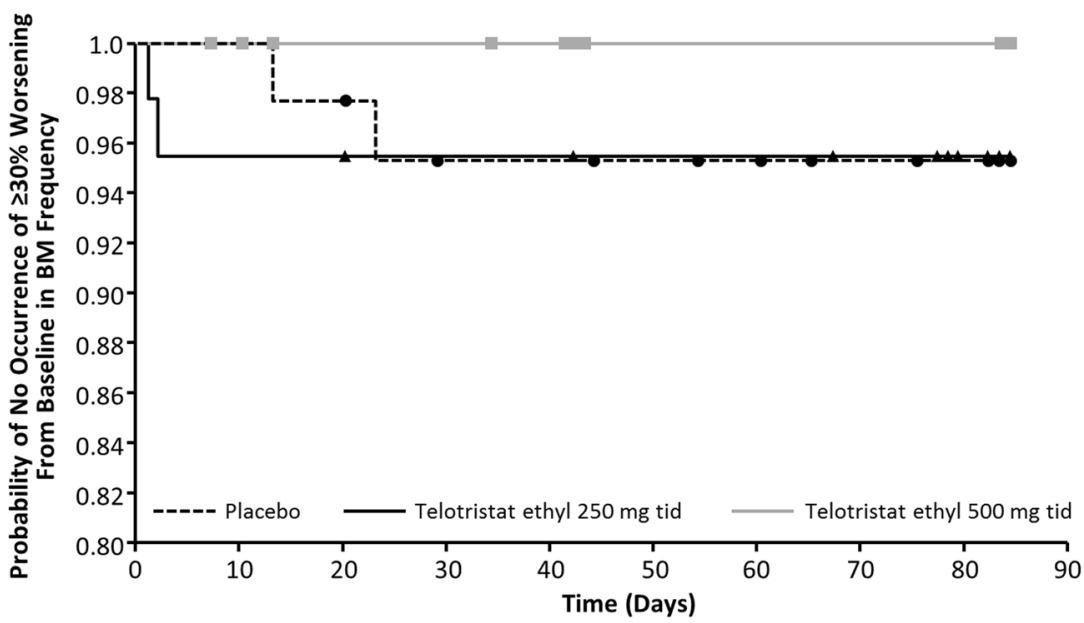

b

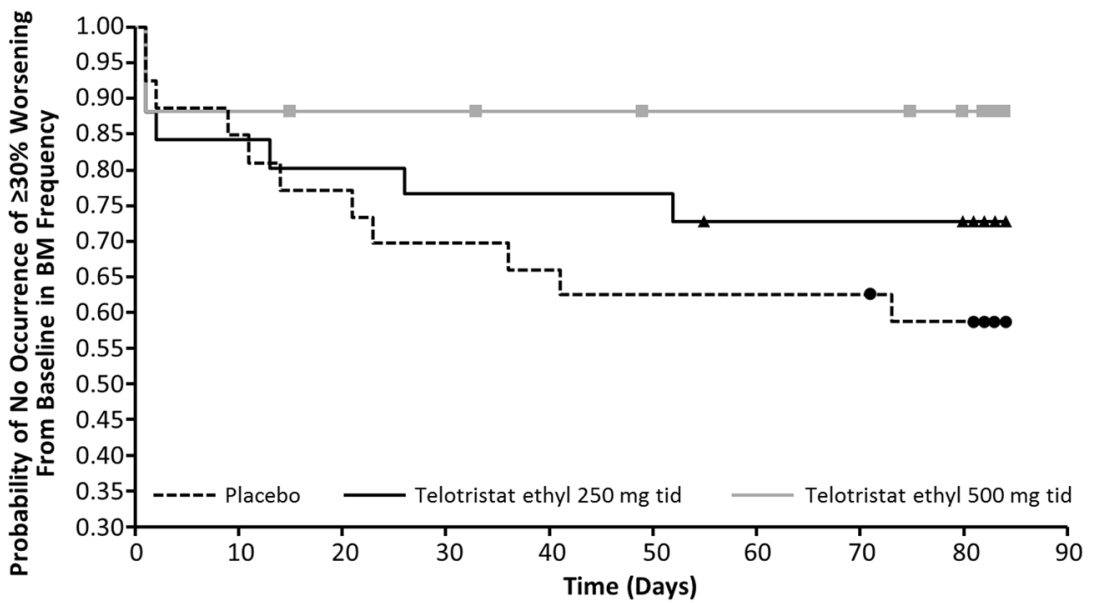

In TELECAST, sustained worsening of BM frequency was more common overall, though fewer patients using telotristat ethyl experienced worsening compared with placebo (SSA alone). During the DBT period, 7 (28\%) patients on telotristat ethyl $250 \mathrm{mg}$ tid, 3 (12\%) patients on telotristat ethyl $500 \mathrm{mg}$ tid, and $11(42 \%)$ patients on placebo had sustained worsening of BM frequency. There was a statistically significant difference between telotristat ethyl $500 \mathrm{mg}$ tid and placebo ( $p=$ 0.021 ). Additionally, in the TELECAST trial, all patients were on concomitant SSA therapy, with the exception of three patients on telotristat ethyl $250 \mathrm{mg}$ tid and five patients on telotristat ethyl $500 \mathrm{mg}$ tid [9].

No median for time to sustained worsening of BM frequency was reached on any treatment arm in TELECAST, although $25 \%$ of patients on placebo experienced sustained worsening by day 21 compared with day 52 for telotristat ethyl $250 \mathrm{mg}$, suggesting a delay in this clinical parameter. Of note, the time to sustained worsening was further delayed on the $500 \mathrm{mg}$ dose of telotristat ethyl (no percentile reached) and is statistically significant $(p=0.021, \mathrm{HR}=0.3[95 \% \mathrm{CI}, 0.06-0.8]$ ) (Fig. 2b).
Stool form was improved in both studies, with the greatest changes observed in the telotristat ethyl $500 \mathrm{mg}$ tid group [8, 9]. Additionally, in TELESTAR, the arithmetic mean change in the proportion of days with urgency to defecate was -0.09 ( $p=0.35$, descriptive) and -0.15 ( $p=0.006$, descriptive) for telotristat ethyl $250 \mathrm{mg}$ tid and telotristat ethyl $500 \mathrm{mg}$ tid, respectively [8]. As both trials reached the median time to sustained improvement in BM frequency by six weeks, data on the mean change from baseline in stool form for patients who experienced $\geq 30 \%$ reduction, $0-30 \%$ reduction, or no reduction in $\mathrm{BM}$ frequency from the TELESTAR and TELECAST studies at six weeks are provided in Fig. $3 a$ and $b$, respectively. In all groups, reductions in BM frequency (when they occurred) were associated with improvements in stool form. Data for the proportion of days with reports of urgency to defecate according to the patients who experienced $\geq 30 \%$ reduction, $0-30 \%$ reduction, or no reduction in $\mathrm{BM}$ frequency in the TELESTAR and TELECAST studies are provided in Fig. $4 a$ and $b$, respectively. 
Fig. 3 Mean change from baseline in stool form at six weeks for patients who experienced $\geq$ $30 \%$ reduction, $0-30 \%$ reduction, or no reduction in baseline BM frequency in the TELESTAR (a) and TELECAST (b) studies. The data from six weeks is presented, as both trials reached the median time to sustained improvement in BM frequency by this point. Abbreviations: BM, bowel movement; SE, standard error; tid, three times per day

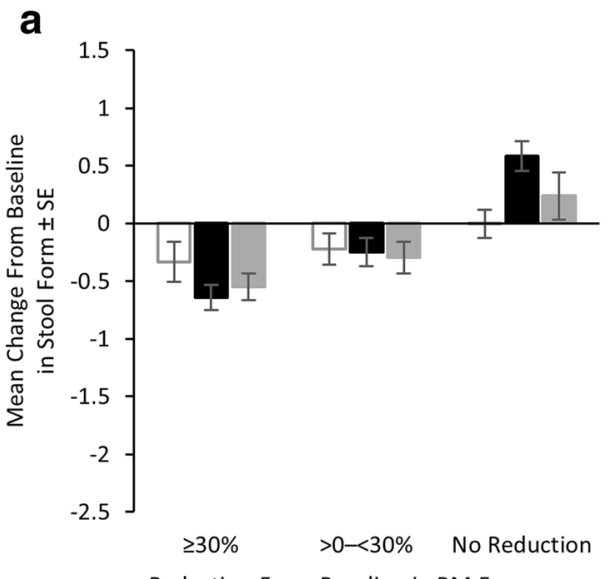

Reduction From Baseline in BM Frequency b

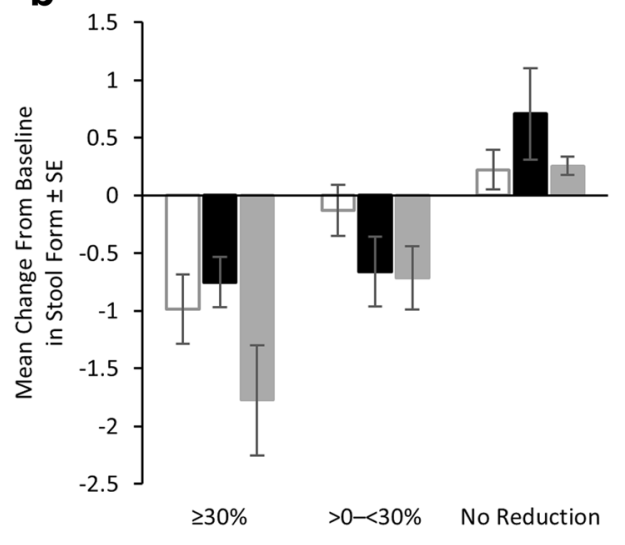

Reduction From Baseline in BM Frequency
Placebo
Telotristat ethyl $250 \mathrm{mg}$ tid
Telotristat ethyl $500 \mathrm{mg}$ tid

\section{Discussion}

Telotristat ethyl is an oral tryptophan hydroxylase inhibitor recently approved in the United States and Europe for the treatment of carcinoid syndrome diarrhea in combination with SSAs, on the basis of efficacy seen in two clinical trials, TELESTAR and TELECAST. Here, the time to sustained $\mathrm{BM}$ frequency reduction or worsening with two dose levels of telotristat ethyl was analyzed versus placebo, with almost all patients taking SSA therapy.

For both trials, median time to sustained BM frequency reduction with telotristat ethyl occurred within 19-39 days, with a range of response time of 3-73 days. In the
TELESTAR study, $\sim 55 \%$ of patients on telotristat ethyl showed a significant reduction in BM frequency by four weeks, $65 \%$ by eight weeks, and $75 \%$ by 12 weeks. Comparatively, BM reductions on placebo were $28 \%, 50 \%$, and $55 \%$, respectively. Given the possibility for late responses to telotristat ethyl, at least eight weeks of therapy may be needed to fully evaluate efficacy.

Sustained worsening in BM frequency was not observed in the TELESTAR study. However, in the TELECAST study, telotristat ethyl was associated with a lower incidence of worsening of BM frequency than placebo, suggesting that some patients may benefit even without a large reduction in BM frequency from
Fig. 4 Proportion of days with reports of urgency/immediate need to defecate at 6 weeks for patients who experienced $\geq 30 \%$ reduction, $0-30 \%$ reduction, or no reduction in baseline BM frequency in the TELESTAR (a) and TELECAST (b) studies. The data from six weeks is presented, as both trials reached the median time to sustained improvement in BM frequency by this point. Abbreviations: BM, bowel movement; SE, standard error; tid, three times per day a

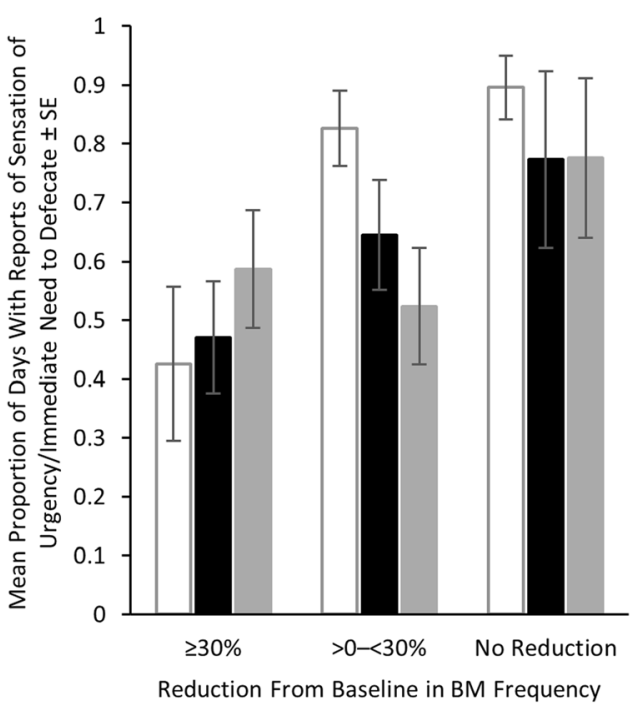

Reduction From Baseline in BM Frequency b

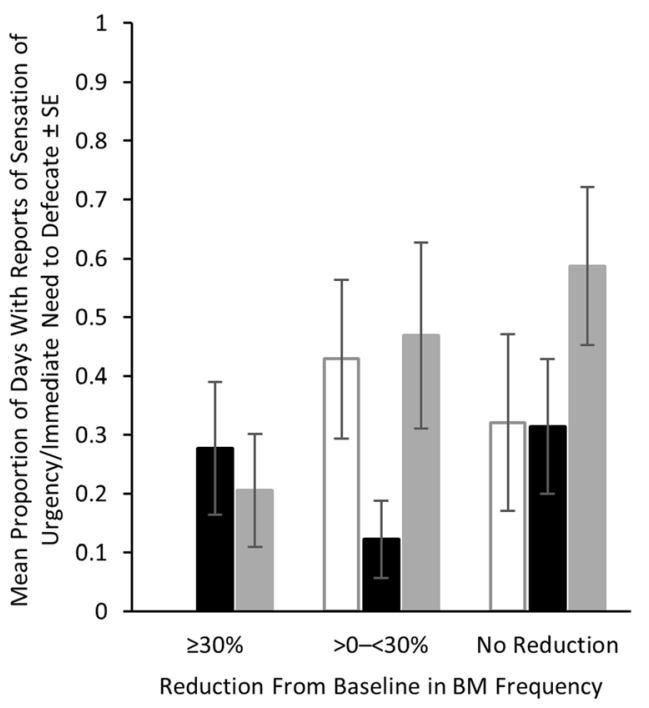

Telotristat ethyl $500 \mathrm{mg}$ tid 
baseline. This may be worth considering for patients with carcinoid syndrome and $<4$ BMs per day.

Other clinical parameters appeared to improve alongside sustained improvement in BM frequency in both trials. Improvements in stool form were observed in both trials, and a reduced proportion of days with an urgency to defecate was observed in TELESTAR $[4,5]$. These outcomes were more marked in the telotristat ethyl $500 \mathrm{mg}$ tid dose level arms, with nonsignificant numerical decreases observed in the telotristat ethyl $250 \mathrm{mg}$ tid arms [4, 5]. In TELESTAR, the 500-mg tid dose level was strongly correlated with reduced u5HIAA levels, reduced average BM frequency, and reduced urgency to defecate [4]. Accordingly, higher baseline BM frequency may be a factor for the improvements observed at this higher dose level, as the TELESTAR study required $\geq 4$ BMs per day as an inclusion criterion [4]. Of note, patients have reported urgency to defecate as one of the most important carcinoid syndrome symptoms to treat, and that carcinoid syndrome symptoms have significant impacts on their lives [18].

The reasons for the wide variability in time to onset of sustained improvement in BM frequency and the substantial numbers of patients who had responses while on placebo are unclear. The difference may relate to the high degree of variability in the systemic absorption between injections of long-acting SSAs. At a highly experienced medical center, only about half the administered SSA injections were successfully targeted to the intramuscular site, resulting in a 3 -fold increase in carcinoid flushing over the following cycle [19]. Variability in the absorption of SSAs in background therapy is an important reason why efficacy is best evaluated over the course of multiple long-acting injections. In both trials, a three- to four-week baseline assessment of BM frequency was used based on the usual dosing frequency for SSAs and the DBT period of 12 weeks in both trials encompassed three or four injections of SSAs. The length of time a patient was on SSA therapy may also play a role, as it is known that patients may become refractory to SSAs over time $[1,11,20]$.

Diet may be another reason for variability in onset of effect and is an important consideration when taking telotristat ethyl for carcinoid syndrome. Dietary composition was not specifically evaluated during these studies. It is recommended that the medication be taken with meals. Administration of telotristat ethyl with a high-fat meal resulted in improved systemic exposure to telotristat ethyl, with a $C_{\max }$ (peak plasma concentration) and $\mathrm{AUC}_{0-\text { inf }}$ (area under the concentration-time curve from 0 to infinity) $112 \%$ and $264 \%$ higher, respectively, compared with the fasted state [5]. A lack of response during initial therapy with telotristat ethyl could potentially be addressed by ensuring that telotristat ethyl is taken with proper meals, and that pancreatic enzymes are used if fat malabsorption is suspected [20]. This point may be especially relevant to patients who limit fat intake because of their carcinoid syndrome.

Patients with diarrhea from causes other than carcinoid syndrome were excluded from these studies. However, diarrhea in these patients is often multifactorial. Factors contributing to diarrhea might include carcinoid-related bioactive compounds (serotonin and others), pancreatic enzyme deficiency, bile acid loss after intestinal surgery, shortened bowel, and bacterial overgrowth. Although it is unclear whether there would be additional patients responding to telotristat ethyl after 12 weeks of therapy, there does seem to be a plateau of onset of effects by that time (approximately $75 \%$ of patients in the TELESTAR study had a sustained response). Some patients are likely to have diarrhea predominantly related to factors other than serotonin produced by NETs, which is the only cause of diarrhea expected to be sensitive to telotristat ethyl. This may account for some of the approximately $30 \%$ of patients who did not seem to respond to telotristat ethyl.

The reliability of the BM frequency measurements was a strength of these studies. Electronic diaries provided time-stamped daily assessments, leading to approximately 10,000 measures of $\mathrm{BM}$ frequency in the TELESTAR study alone. This allowed an ample basis for careful review of time patterns. A key limitation of the studies was the inability to comprehensively examine all the different variables that can affect BM frequency in clinical trials. For instance, neither diet nor individual doses of over-the-counter diarrhea treatments were tracked on a daily basis. Short-acting octreotide use was examined, and its use was reduced on telotristat ethyl compared with placebo (SSA alone) in the TELESTAR study [8]. More information on these and other variables could potentially help maximize efficacy.

In conclusion, sustained reductions of at least $30 \%$ in BM frequency on telotristat ethyl were observed within 3 to 6 weeks in half of the patients in these phase III trials with a range of onset between three and 73 days. Results of the trials suggest that some patients on telotristat ethyl may also have benefited by avoiding a worsening in $\mathrm{BM}$ frequency. Additionally, the telotristat ethyl dose level correlated with improvements in stool form and urgency. These parameters were generally associated with BM frequency and may help in the evaluation of telotristat ethyl efficacy. Understanding the time course of telotristat ethyl effects may help ensure that patients and their care providers have appropriate 
expectations and guide the initial assessment of the drug in patients with carcinoid syndrome diarrhea.

Acknowledgments We thank the patients and investigators for participating in the studies. We thank James Banigan, PhD (Chameleon Communications International, with funding provided by Lexicon Pharmaceuticals, Inc.) for medical editorial assistance with this manuscript. We thank the following Lexicon employees: Shanna Jackson, RN, MBA, JD Wallace, Karie Arnold, Nam Womack, Kristi Boehm, MS, ELS, and Rosanna Fleming, MS. We would like to thank the team at INC Research (Raleigh, NC) for study conduct, monitoring, analysis, and reporting. Lastly, we thank Ipsen Pharmaceuticals, Inc., a partner of Lexicon Pharmaceuticals, Inc., for review of this manuscript for medical accuracy.

Funding Information This work was supported by Lexicon Pharmaceuticals, Inc., The Woodlands, TX, USA. Employees of the company were involved in the study designs; the collection, analysis, and interpretation of data; the writing and review of the manuscript; and the decision to submit for publication.

\section{Compliance with Ethical Standards}

Conflict of Interest Joseph S. Dillon: Lexicon Pharmaceuticals, Inc. (RF); Matthew H. Kulke: Lexicon Pharmaceuticals, Inc. (C/A), Ipsen Bioscience (C/A), Novartis Pharmaceuticals (C/A); Dieter Hörsch: Lexicon Pharmaceuticals, Inc. (H), Ipsen Pharmaceuticals, Inc. (RF, H); Lowell B. Anthony: Lexicon Pharmaceuticals, Inc. (RF, H); Richard R. P. Warner: Lexicon Pharmaceuticals, Inc. (RF); Emily Bergsland: UpToDate (IP), Novartis (RF); Staffan Welin: Ipsen (C/A), Novartis (C/A); Pamela L. Kunz: Lexicon Pharmaceuticals, Inc. (C/A), Advanced Accelerator Applications (RF), Genentech (RF), Ipsen (RF), Merck (RF), Incyte (RF); Chad McKee: Lexicon Pharmaceuticals, Inc. (E, OI); Pablo Lapuerta: Lexicon Pharmaceuticals, Inc. (E, OI); Phillip Banks: Lexicon Pharmaceuticals, Inc. (E, OI); Marianne Pavel: Novartis Pharmaceuticals (RF, H), Ipsen (RF, H), Lexicon Pharmaceuticals, Inc. $(\mathrm{H})$, Pfizer, Inc. $(\mathrm{H})$. Thomas M. O'Dorisio indicated no financial relationships.

(C/A) Consulting/advisory relationship; (RF) Research funding; (E) Employment; (ET) Expert testimony; (H) Honoraria received; (OI) Ownership interests; (IP) Intellectual property rights/inventor/patent holder; (SAB) Scientific advisory board.

Ethical Approval All procedures performed in studies involving human participants were in accordance with the ethical standards of the institutional and/or national research committee and with the 1964 Helsinki declaration and its later amendments or comparable ethical standards. This article does not contain any studies with animals performed by any of the authors.

Informed Consent Informed consent was obtained from all individual participants included in the study.

Open Access This article is licensed under a Creative Commons Attribution 4.0 International License, which permits use, sharing, adaptation, distribution and reproduction in any medium or format, as long as you give appropriate credit to the original author(s) and the source, provide a link to the Creative Commons licence, and indicate if changes were made. The images or other third party material in this article are included in the article's Creative Commons licence, unless indicated otherwise in a credit line to the material. If material is not included in the article's Creative Commons licence and your intended use is not permitted by statutory regulation or exceeds the permitted use, you will need to obtain permission directly from the copyright holder. To view a copy of this licence, visit http://creativecommons.org/licenses/by/4.0/.

\section{References}

1. Ruszniewski P, Ish-Shalom S, Wymenga M, O'Toole D, Arnold R, Tomassetti P, et al. Rapid and sustained relief from the symptoms of carcinoid syndrome: results from an open 6-month study of the 28day prolonged-release formulation of lanreotide. Neuroendocrinology. 2004;80:244-51.

2. Beaumont JL, Cella D, Phan AT, Choi S, Liu Z, Yao JC. Comparison of health-related quality of life in patients with neuroendocrine tumors with quality of life in the general US population. Pancreas. 2012;41:461-6.

3. Janson ET, Oberg K. Long-term management of the carcinoid syndrome. Treatment with octreotide alone and in combination with alpha-interferon. Acta Oncol. 1993;32:225-9.

4. Toumpanakis C, Garland J, Marelli L, Srirajaskanthan R, Soh J, Davies $\mathrm{P}$, et al. Long-term results of patients with malignant carcinoid syndrome receiving octreotide LAR. Aliment Pharmacol Ther. 2009;30:733-40.

5. Lexicon Pharmaceuticals Inc. Xermelo® (telotristat ethyl) [prescribing information]. Lexicon Pharmaceuticals, Inc., The Woodlands, TX, 2017. Available at https://www.accessdata.fda. gov/drugsatfda_docs/label/2017/208794s000lbl.pdf. Accessed February 20, 2017.

6. European Commission. Commission implementing decision of 18.9.2017 granting marketing authorisation under regulation (EC) No 726/2004 of the European Parliament and of the Council for "xermelo - telotristat", an orphan medicinal product for human use. 2017. Available at http://ec.europa.eu/health/documents/ community-register/2017/20170918138652/dec 138652 en.pdf. Accessed 2 Oct 2017.

7. National Comprehensive Cancer Network. Neuroendocrine tumors and adrenal tumors (version 1.2018). 2018. Available at https:// www.nccn.org/professionals/physician_gls/pdf/neuroendocrine. pdf. Accessed 30 March 2018.

8. Kulke MH, Horsch D, Caplin ME, et al. Telotristat ethyl, a tryptophan hydroxylase inhibitor for the treatment of carcinoid syndrome. J Clin Oncol. 2017;35:14-23.

9. Pavel ME, Gross DJ, Benavent M, Perros P, Srirajaskanthan R, Warner RRP, et al. Telotristat ethyl in carcinoid syndrome: safety and efficacy in the TELECAST phase 3 trial. Endocr Relat Cancer. 2018;25:309-22.

10. Zandee WT, Kamp K, van Adrichem RC, Feelders RA, de Herder WW. Effect of hormone secretory syndromes on neuroendocrine tumor prognosis. Endocr Relat Cancer. 2017;24:R261-74.

11. Alonso-Gordoa T, Capdevila J, Grande E. GEP-NETs update: biotherapy for neuroendocrine tumours. Eur J Endocrinol. 2015;172:R31-46.

12. Molina-Cerrillo J, Alonso-Gordoa T, Martinez-Saez O, et al. Inhibition of peripheral synthesis of serotonin as a new target in neuroendocrine tumors. Oncologist. 2016;21:701-7.

13. Gelhorn HL, Kulke MH, O'Dorisio T, Yang QM, Jackson J, Jackson S, et al. Patient-reported symptom experiences in patients with carcinoid syndrome after participation in a study of telotristat etiprate: a qualitative interview approach. Clin Ther. 2016;38:75968.

14. Kulke MH, O'Dorisio T, Phan A, Bergsland E, Law L, Banks P, et al. Telotristat etiprate, a novel serotonin synthesis inhibitor, in patients with carcinoid syndrome and diarrhea not adequately controlled by octreotide. Endocr Relat Cancer. 2014;21:705-14.

15. Lewis SJ, Heaton KW. Stool form scale as a useful guide to intestinal transit time. Scand J Gastroenterol. 1997;32:920-4.

16. Longstreth GF, Thompson WG, Chey WD, Houghton LA, Mearin F, Spiller RC. Functional bowel disorders. Gastroenterology. 2006;130:1480-91.

17. Data on file. Lexicon Pharmaceuticals, Inc, Princeton, NJ. 
18. Anthony L, Ervin C, Lapuerta P, Kulke MH, Kunz P, Bergsland E, et al. Understanding the patient experience with carcinoid syndrome: exit interviews from a randomized, placebo-controlled study of telotristat ethyl. Clin Ther. 2017;39:2158-68.

19. Boyd AE, DeFord LL, Mares JE, Leary CC, Garris JL, Dagohoy $\mathrm{CG}$, et al. Improving the success rate of gluteal intramuscular injections. Pancreas. 2013;42:878-82.
20. Strosberg JR, Halfdanarson TR, Bellizzi AM, Chan JA, Dillon JS, Heaney AP, et al. The North American Neuroendocrine Tumor Society consensus guidelines for surveillance and medical management of midgut neuroendocrine tumors. Pancreas. 2017;46:707-14.

Publisher's Note Springer Nature remains neutral with regard to jurisdictional claims in published maps and institutional affiliations. 